\title{
Managing the impact on the electric distribution network of rapid of electric vehicle adoption
}

\author{
Chuck Roy ${ }^{1}$ \\ ${ }^{\text {I} C h u c k ~ R o y, ~ S i e m e n s ~ S m a r t ~ G r i d ~ S e r v i c e s, ~ H o u s t o n, ~ T X, ~ c h a r l e s . r o y @ s i e m e n s . c o m ~}$
}

\begin{abstract}
Many electric utilities have begun piloting Electric Vehicle (EV) charging stations with a number of manufacturers, including Siemens. As a result of working with these EV charging station manufacturers, utilities have developed a good understanding of prevailing technology. While utilities consider options like selling or recommending preferred EV charging stations to new EV owners in their service territory, a trend has emerged where new EV owners purchase charging station technology without notifying the utility based on recommendations from their EV dealers. It is important for the utilities to be aware of customers in their service territories that are installing EV charging stations in their homes and businesses in order to plan for the required distribution network upgrades to serve these stations.
\end{abstract}

Siemens has worked with a number of utilities to discuss their business requirements for a software solution envisioned to manage the installation and use of EV charging stations in their networks. This paper reviews both the problem these utilities are trying to solve together with their functional and integration requirements for a comprehensive EV charging station management system which can support utility programs including critical peak pricing programs, demand response, outage management, etc.

Keywords: electric vehicle, charging stations, electric utility distribution systems

\section{Introduction}

The US Department of Energy has forecasted that 100 million electric or hybrid cars, or $40 \%$ of domestic automobiles, will be in use by 2020 . Studies from Berkley go further and project $64 \%$ of domestic automobiles will be electric by 2030 . The challenge to electric utilities is to build out an electric distribution infrastructure capable of delivering energy to this new fleet of vehicles in the next 10 years.

A typical electric utility distribution system delivers power to a residential EV charging station from a transformer which typically serves 6 customers by stepping down the utility medium voltage to dual 120Vac single phase (also called 240 Vac split phase). This voltage is fed through a meter into the residential load control centre. The load control centre consists of branch circuit breakers and distributes the power supply within various areas within the home.

An electric vehicle can connect to the load control centre directly through a domestic wall socket (a.k.a. "Mode 1") where it may share the circuit with other load in the home or indirectly through 
an EV charging station, installed by an electrician, on a dedicated branch circuit (Mode 2 or Mode 3).

According to Mike Tinskey, manager of sustainability activities at Ford Motor Company (F-NYSE),

"That [electric] vehicle is going to be the largest discretionary load on the home. What if you had a neighbourhood with 10 electric vehicles and they're all on the same transformer? That's a real problem for utilities, and they want to be able to manage that."

Although most electric vehicles support charging from a domestic wall socket, the benefits of using a dedicated EV charge station include faster charging times and sensing mechanisms to disconnect the power when the EV has completed charging.

Typical electric vehicles have a battery capacity of $20 \mathrm{kWh}$ and a range of $150 \mathrm{~km}$. Charging times vary based on the charging mode. The International Electrotechnical Commission (IEC) has categorized different types of charging modes as:

Mode 1 - slow charging from a regular single or 3-phase electrical socket using standard current (typically 10 amps). Charge times typically take 10 hours.

Mode 2 - slow charging from a regular socket equipped with an EV-specific earthing cable which has built in protection. Charge times typically take 8 hours.

Mode 3 - slow or fast charging using a specific EV multi-pin socket with control and protection functions (e.g. SAE J1772 and IEC 62196). Charge times typically take 4 hours,

Mode 4 - Direct current (DC) fast charging using some special charger technology such as CHAdeMO. Charging times typically take 20 minutes.

Most EV charge stations support "Mode 3" charging. Residential EV charging station units are typically "dumb" chargers that don't authenticate the user, deny access to EV car owner to prevent charging nor communicate with a host (i.e. they are not networked devices).

\section{Challenges facing Electric Utilities}

A poorly managed, rapid introduction of EV charging stations in the utility's service territory will trigger expensive, unplanned electric distribution network upgrades to mitigate power quality issues and power outages related to the stations. EV charging stations will also contribute to an increase in peak demand and create issues with "cold load" pick-up procedures during outage restoration. Specific affects include:

- Electric utility customers will suffer more frequent power outages,

- Electric distribution operations will need to react to unplanned network upgrades

- Call centre personnel will experience a higher number of complaints.

These problems will not only result in increased customer dissatisfaction, but also create a perception that the utility can't handle the roll-out of EV cars which could, in turn, adversely impact EV adoption rates. Issues with cold load pick-up also result in increased customer minutes of interruption and adversely impact related indices like SAIDI, CAIFI, etc.

\section{Electric Utility Requirements}

. Working with utilities, Siemens identified a number of critical functional requirements for a new software solution designed to manage the EV charge stations and their deployment. Functional requirements for the $\mathrm{EV}$ charge station management system (CSMS) include:

R1: Tracking the physical and electrical address of new EV charge stations as they are added to the electric distribution network.

R2: For willing EV charge stations owners, managing EV charging schedules

R3: For willing EV charge stations owners, enrolling the EV charging stations in a Demand Response (DR) program.

R4: Collection of EV charging data to monitor usage in the context of schedule and DR event participation.

Given these functional requirements, the following technical requirements emerged:

R5: A server-side software solution to keep track of, and communicate with, all the EV charging 
stations and optional charge station controllers designed to manage "dumb" charging stations.

R6: Support for communication with the EV charge stations through the either the utility's AMI network or Cellular network with a residential local Home Area Network (HAN) communication protocol (e.g. ZigBee SEP 1.x, 2.x).

\section{Envisioned Solution}

A software system that can coordinate EV charging sessions and potentially control other discretionary loads across the utility service territory with the cooperation of EV owners would mitigate the need for unplanned upgrades to the electric distribution network and related increases in utility operating budget as well as reduce the risk of overloading the network.

Working with select utilities, Siemens elaborated the requirements above to develop a simple Business Use Case Diagram, for the CSMS to describe how such a system could manage and enforce the EV charging schedules through communication with the charging station controllers via an EV "head-end" communication network by enabling and disabling the EV charge stations. The envisioned system could be used to manage Demand Response (DR) Events and collect the EV charge data to confirm EV schedule and DR event execution.

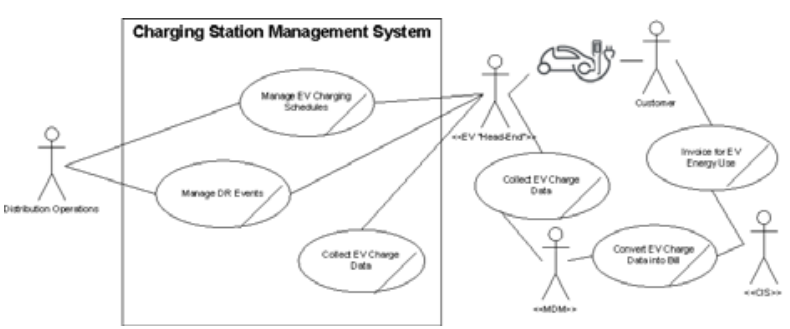

Figure1: CSMS Business Use Case Diagram

Per the illustration above, the EV "head-end" would be responsible for collecting EV usage data, through direct communication with the stations, and for transmitting this usage data to the CSMS as well as for processing by the MDM and CIS.

This model invites existing smart charging station manufacturers, who have already networked their stations, to integrate with the utility CSMS.

\section{Benefits}

Rolling out an EV charge station management system that meets the business requirements described above will help the utility manage the rapid deployment of EV charge stations in their territory. The system would support the sale (or registry of) EV charging stations to owners to help utilities manage the deployment of the stations. Through promotional rates, utilities can not only register the stations but also work with station owners to sub-meter the stations and manage and control the charging sessions of each station proactively with the CSMS.

\section{Conclusion}

Utilities that take a proactive role in the registration and management EV charging stations in their service territories with a solution like CSMS are better prepared to manage distribution network upgrades if the adoption rates for charging stations increase dramatically. Compensating charge station owners through targeting pricing programs to allow the utilities to set charging schedules coupled with DR would allow the utility to have the necessary control over the charging sessions to directly address power quality issues related to concurrent EV charging sessions.

\section{References}

[1] Stephen Marcus (February 15, 2010). "Contenders vie for quickest EV battery charging time" (http://cleantech.com/news/5628/quickest-evbatteryrecharge-fast) . Cleantech Group.

[3] Roy C. "Charge Station Management System \& Charge Station Controllers Business Requirements for Duke Energy" Siemens Energy, Inc. - Nov/2011

[4] Brooks G. "Coming to Your Neighbourhood: Electric Car Surcharges?”. September 14, 2010, issue

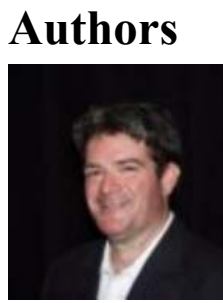

Chuck Roy, Sr. Engineering Manager for Siemens Smart Grid Services based in Houston, TX has been engineering and delivering distribution \& transmission software solutions to electric utilities since 1991. 